\title{
Una metodología para evaluar el manejo del turismo en áreas naturales protegidas
}

\author{
Jorge Mario Chávez Salas ${ }^{\mathrm{a}}$, Alexandre França Tetto ${ }^{\mathrm{b}}$, Carlos Augusto Reynel ${ }^{\mathrm{a}}$, Nilton José Sousa ${ }^{\mathrm{b}}$, \\ Andressa Tres ${ }^{\mathrm{b}}$, Mariana Meira Micaloski ${ }^{\mathrm{b}^{*}}$, Gonzalo Javier Oliveira Flores ${ }^{\mathrm{b}}$
}

${ }^{\text {a } U n i v e r s i d a d e ~ N a c i o n a l ~ A g r a ́ r i a ~ d a ~ M o l i n a, ~ P e r u ~}$
b Universidade Federal do Paraná (UFPR), Brasil
${ }^{*}$ Autor correspondente (micaloskimariana@ gmail.com)

\section{N F O}

\section{Keywords}

governance sustainability protected areas sustainable

\section{Palabras-clave}

gobernanza sostenibilidad áreas protegidas turismo sostenible

\begin{abstract}
A B S T R A C T
A methodology to evaluate the management of tourism in protected natural areas

There are several methodologies to evaluate the management of protected areas, but there are no references focused exclusively on tourism activity. Therefore, this research proposes a methodology to determine the tourism management in individual protected areas. For this, six international documents related to tourism, nature and culture were reviewed, and six criteria were obtained to assess the tourism management in protected natural areas. Interviews were conducted with 24 specialists from the tourism and protected natural areas and information was obtained to determine the variables to be evaluated within the six determined criteria. A first evaluation matrix was developed with a numerical evaluation system to be applied to the criteria and variables, which was presented and discussed in a validation workshop with 14 specialists. Finally, with the comments and suggestions obtained, the refinement of the matrix and the numerical evaluation was carried out, with which 56 variables were obtained to be analyzed for the six criteria. Each of the variables was assigned four levels of qualification. Using scores and comparative graphs, it is possible to determine which are the criteria that should be worked the most and, within them, which are the strongest or weakest variables, to apply greater efforts in their development. This methodology is expected to contribute to the systematic development of tourism in protected natural areas, under the concepts of sustainability.
\end{abstract}

\section{R E S U M E N}

Existen diversas metodologías para evaluar la gestión de áreas naturales protegidas, pero no se encuentran referencias enfocadas exclusivamente a la actividad turística. Por eso, esta investigación propone una metodología para determinar los avances del manejo de turismo en áreas naturales protegidas individuales. Para su diseño, se revisaron seis documentos internacionales relacionados con el turismo, la naturaleza y la cultura, y se obtuvieron seis criterios para evaluar los avances del manejo del turismo en las áreas naturales protegidas. Se preparó un programa de entrevistas a 24 especialistas en el área del turismo y áreas naturales protegidas y se obtuvo información para determinar las variables a evaluar dentro de los seis criterios determinados. Se desarrolló una primera matriz de evaluación con un sistema de evaluación numérica a aplicarse a los criterios y variables, que fue presentada y discutida en un taller de validación con 14 especialistas. Finalmente, con los comentarios y sugerencias obtenidos, se realizó el afinamiento de la matriz y de la evaluación numérica, con lo que se obtuvieron 56 variables a ser analizadas para los seis criterios. A cada una de las variables se le asignaron cuatro niveles de calificación. Usando los puntajes y las gráficas comparativas, se puede determinar cuáles son los criterios que más deben ser trabajados y, dentro de ellos, cuáles son las variables más sólidas o más débiles, para aplicar mayores esfuerzos en su desarrollo. Se espera que esta metodología contribuya al desarrollo sistemático del turismo en las áreas naturales protegidas, bajo los conceptos de sostenibilidad. 


\section{INTRODUCCIÓN}

Las áreas naturales protegidas son un elemento clave para cualquier iniciativa o estrategia mundial cuya finalidad sea la conservación de la naturaleza. Si bien la generación de ingresos económicos por concepto de pago de entradas a las áreas naturales protegidas es importante (muchas veces la presencia de visitantes depende de la belleza de los paisajes, elementos de flora y fauna saludables, posibilidades de actividades atractivas y presencia de culturas auténticas) y beneficia directa e indirectamente a personas y empresas locales. El turismo contribuye a fortalecer la conexión de los visitantes con los valores de las áreas naturales protegidas, lo que ayudará a su sensibilización en relación con la conservación del patrimonio que estas involucran.

El turismo en las áreas naturales protegidas es un componente importante en la industria turística global, pues genera empleos y tiene impactos positivos en la economía, la sociedad y la cultura. Al ser una de las mayores actividades económicas del mundo, es preciso que tenga una infraestructura adecuada para proteger el ambiente. Si las actividades turísticas son mal manejadas o inapropiadas, sus impactos negativos pueden ser muy amplios, lo que afectaría los recursos de las áreas protegidas, las economías y comunidades locales y a los mismos turistas (Leung et al., 2019).

Existen diversas metodologías para evaluar el manejo integral de áreas naturales protegidas como por ejemplo los estudios de efectividad de manejo (PAME) que comenzaron a diseñarse en la década de 1990 por la administración de algunas áreas protegidas $\mathrm{u}$ organizaciones internacionales no gubernamentales enfocadas en la conservación de la naturaleza, y que han continuado a lo largo del presente siglo (Rivero Blanco \& Gabaldón, 1992; Hockings et al., 2006; 2007). Estas definen la evaluación de la efectividad del manejo del área, y principalmente, en qué medida se están conservando valores y logrando metas y objetivos. Sin embargo, no se hallan reportes de una metodología para evaluar específicamente una de las actividades que mayor impacto, positivo o negativo, puede tener en un área natural protegida: el manejo del turismo.

El objetivo de esta investigación es desarrollar una metodología que contribuya a la evaluación del manejo del turismo en áreas protegidas, utilizando un sistema de puntajes de criterios y variables que cubran los diversos elementos que implican esta actividad en las áreas. Además de ayudar al manejo del turismo, se busca contribuir a planificar mejor las actividades turísticas sostenibles en áreas que actualmente cuentan con visitantes y en otras con potencial para desarrollar la actividad.

\section{MATERIALES E MÉTODOS}

El trabajo de investigación utiliza un enfoque cualitativo a partir de diferentes metodologías de investigación social y ha sido desarrollado en cuatro etapas (figura 1).

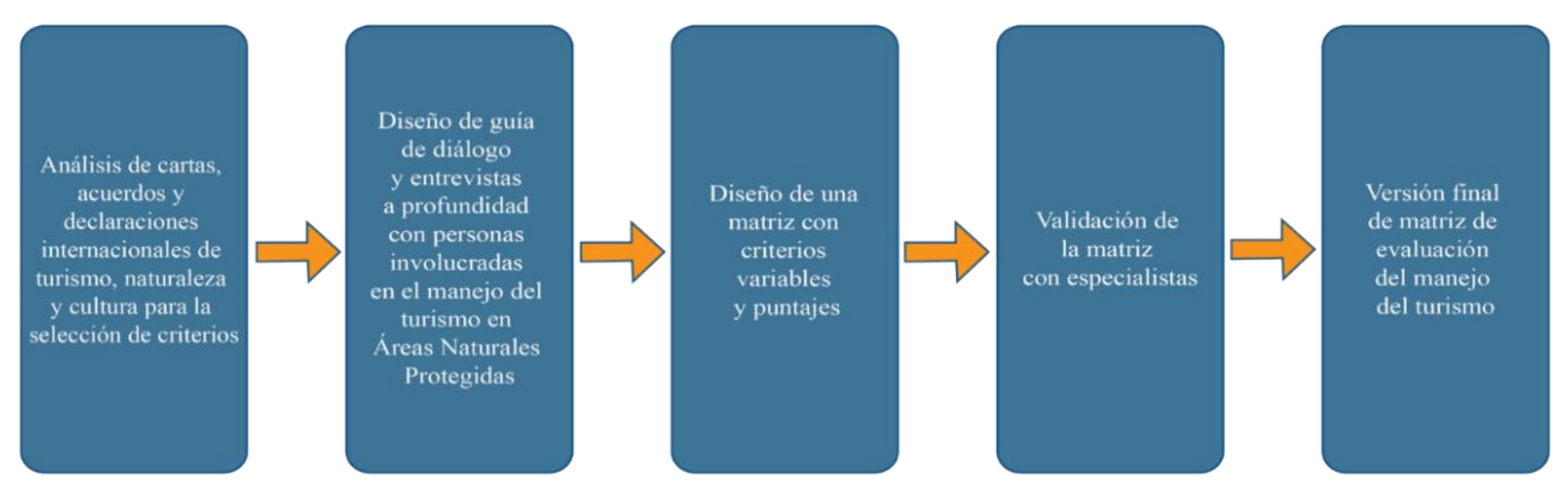

Figura 01 - Síntesis de la metodología desarrollada para evaluar el manejo del turismo en un área natural protegida.

La primera etapa fue el análisis de seis declaraciones, códigos y cartas internacionales relacionado con el turismo sostenible, la naturaleza y la cultura, con el fin de identificar los criterios más relevantes para la evaluación del manejo turístico en los sistemas de áreas naturales. Estos documentos de relevancia internacional se han convertido en una herramienta de discusión, negociación, cooperación y compromiso, que ha permitido generar normativa que recupere las particularidades de las partes para tratar problemáticas globales (Rodríguez, 2009). Para seleccionarlos, se determinó que cumpliesen las siguientes condiciones: (i) el evento que diese como consecuencia el documento debía 
estar organizado por instituciones de reconocimiento global; (ii) en los documentos debía prevalecer el turismo, naturaleza y cultura; (iii) el carácter declarativo de los documentos; (iv) el valor consensual, tanto en los eventos donde fueron producidos como a posteriori en la comunidad académica; (v) el valor referencial resumen en materia de las buenas prácticas en la gestión turística.

Los seis documentos seleccionados fueron: la Carta del Turismo Sostenible (Conferencia Mundial de Turismo Sostenible, 1995); la Carta de Málaga sobre turismo justo (Encuentro Internacional sobre Turismo Justo, 2006); la Declaración de Ciudad del Cabo sobre el turismo responsable (International Conference on Responsible Tourism in Destinations, 2002); la Declaración de Muscat sobre Turismo y Cultura (Organización Mundial del Turismo et al., 2017); el código ético ambiental para el turismo (Organización Mundial del Turismo, 1999) y la Declaración de Quebec sobre el ecoturismo (Programa de las Naciones Unidas para el Medio Ambiente \& Organización Mundial del Turismo, 2002).

Una vez elegidos los documentos, se revisaron a profundidad, anotando los criterios que tenían más incidencia en la siguiente secuencia: (i) identificación de las palabras claves comunes a los documentos analizados; (ii) verificación de la pertinencia texto-contexto en cada documento; (iii) determinación de la superposición y complementariedad de los epígrafes; (iv) formulación de la matriz documental resumen.

Después de la formulación de la matriz del documento, se fueron agrupando los criterios y al final quedaron seleccionados seis criterios que serán la base para el trabajo de las siguientes etapas. El uso de matrices trabaja como un mecanismo que asiste la medición de parámetros y facilita tanto la evaluación como el seguimiento del comportamiento de sistemas en diversos marcos de referencia (Chirino et al., 2008).

La segunda etapa comprendió el diseño de una guía de diálogo para ser aplicada en entrevistas a profundidad a personas relacionadas con turismo, naturaleza y cultura. Utilizando los seis criterios obtenidos en la etapa anterior, se plantearon cuestiones vinculadas con dichos aspectos con el propósito de obtener información que ayude a estructurar la matriz de evaluación. La estructuración de los tópicos abordados en la conversación tuvo como objetivo ayudar a la conducción de las entrevistas para que el entrevistador tuviese la libertad de desarrollar cada situación con la flexibilidad suficiente, permitiendo explorar más ampliamente una cuestión. Así, el guion logró orientar a los entrevistados en los cuestionamientos. El propósito de ese tipo de entrevista es obtener del entrevistado lo que él piensa sobre el tema, los aspectos más relevantes de determinado problema y otras opiniones propias del entrevistado. De esa forma, las entrevistas sirvieron de apoyo en la comprobación de los resultados de las fuentes documentales (Biesek, 2013).

Las entrevistas se efectuaron directa y personalmente a 24 personas con amplio conocimiento y experiencia sobre los temas de turismo, áreas naturales protegidas, naturaleza y cultura, tanto del sector público como del privado. Para la elaboración del reporte de entrevistas se tomaron en cuenta los criterios universalmente válidos para los estudios cualitativos (enfoque inductivo, técnicas no estructuradas, flexibilidad, data narrativa y análisis interpretativo) y se ha desarrollado la secuencia metodológica que se describe a continuación: formulación de la guía de diálogo, identificación de las ideas principales, determinación de coincidencias y discrepancias y obtención de conclusiones e inferencias. El producto de la segunda etapa fue la sistematización de las respuestas obtenidas a partir de las respuestas a las preguntas formuladas, clasificadas en función de los seis criterios identificados en la primera etapa.

La tercera etapa consistió en el diseño de un modelo de evaluación del manejo del turismo en el área natural protegida. El objetivo de esta etapa fue contar con una matriz base de evaluación de los avances del manejo de turismo en un área natural protegida. Se buscó que la matriz pueda cumplir con las siguientes condiciones: ser fácilmente replicable, capaz de suministrar datos coherentes para permitir el seguimiento de los avances en el tiempo y una "puntuación" del área protegida y ser de fácil entendimiento (Ministerio del Ambiente del Ecuador, 2014). Dado que no hallaron en la bibliografía consultada investigaciones que incluyan matrices de evaluación del manejo turístico en áreas naturales protegidas, se utilizaron como referencias diversos trabajos de efectividad de manejo e indicadores en áreas protegidas, como por ejemplo Leverington et al. (2010), que han hecho un estudio global de evaluación de la eficiencia del manejo en áreas protegidas; Hockings et al. (2006), que realizaron un marco para evaluar la eficiencia del manejo en área protegidas; Stolton et al. (2007), quienes desarrollaron una herramienta de monitoreo de la efectividad del manejo en áreas naturales protegidas; Worboys et al. (2015), quienes en su publicación dedican a profundidad un capítulo al turismo; Sancho et al. (2007), que trabajaron con indicadores de sostenibilidad para diversos destinos; Sánchez-Cortez et al. (2014), que realizaron una propuesta de matriz para evaluación de proyectos geoparques en América Latina con base en ANP; y el Ministerio del Ambiente del Ecuador (2014), que desarrolló una 
guía metodológica para la evaluación de efectividad de manejo del patrimonio de áreas naturales del Estado.

La matriz de evaluación del manejo del turismo consistió en asignar un diverso número de variables a los seis criterios trabajados en la primera y segunda etapas de la investigación, buscando tener una cobertura total de las condiciones a cubrir para un manejo pertinente del turismo en cada área. A cada variable se le asignaron cuatro niveles que pudiesen graficar en la mejor medida lo avanzado por la administración del área. Las variables fueron seleccionadas en función de la revisión bibliográfica y las entrevistas sostenidas con los especialistas. Después de haberse planteado las variables, se analizaron opciones para otorgar puntajes que ayuden a simplificar la evaluación. Se eligió un sistema de puntos de $0,1,2$ y 3 según el grado de avance de cada variable y luego se plantearon medios de comparación. La matriz diseñada ha buscado incorporar el mayor número posible de elementos para evaluar, a fin de contar con mejores elementos de juicio, pero cabe señalar que puede cambiar en número de variables (aumentar o disminuir) si se cree conveniente, dado el carácter especial de cada área natural protegida. La fórmula planteada permite hacer cambios en el cálculo de los promedios.

La cuarta etapa consistió en la validación de la matriz diseñada, a través de un taller con especialistas a partir de los siguientes pasos que plantean
Weiskopf e Landero (2009): (i) selección de especialistas en el tema de turismo, áreas naturales protegidas, planificación y servicios turísticos: se revisaron publicaciones y se hicieron consultas a los gremios para seleccionar los invitados al taller; (ii) invitación a los especialistas, adjuntándoles vía electrónica la matriz con una semana de anticipación para su familiarización previa; (iii) taller con la siguiente agenda: registro de invitados, agradecimiento y explicación de motivos del taller, presentación audiovisual del taller, debate de los temas, anotación de sugerencias y cierre del taller. Finalmente, se volcaron los comentarios y sugerencias de cambios realizadas por los participantes hasta obtener la versión final de la matriz de evaluación del manejo del turismo en un área natural protegida.

\section{RESULTADOS Y DISCUSIÓN}

Los seis documentos que cumplían las condiciones estipuladas en la metodología de la etapa 1se presentan en la tabla 1.

De la revisión a profundidad de los documentos, se obtuvieron los seis criterios que se presentan a continuación: gobernanza de la gestión, conservación del patrimonio, identidad cultural y autenticidad, inclusión y equidad, mercadeo y comercialización y experiencia del visitante.

Tabla 01 - Características de los documentos internacionales analizados.

\begin{tabular}{lllll}
\hline \multicolumn{1}{c}{ Fuente } & \multicolumn{1}{c}{ Evento } & Promotores & \multicolumn{1}{c}{ Locación } & \multicolumn{1}{c}{ Objetivo } \\
\hline $\begin{array}{l}\text { Carta del turismo } \\
\text { sostenible }\end{array}$ & $\begin{array}{l}\text { Conferencia Mundial } \\
\text { del Turismo Sosteni- } \\
\text { ble, } 1995\end{array}$ & $\begin{array}{l}\text { UNESCO, } \\
\text { PNUMA, OMT }\end{array}$ & $\begin{array}{l}\text { Lanzarote, Is- } \\
\text { las Canarias, } \\
\text { España }\end{array}$ & $\begin{array}{l}\text { Establecer un importante cambio de rumbo en la } \\
\text { industria del turismo y en el desarrollo de esta ac- } \\
\text { tividad, incluyendo los propios destinos, hacia } \\
\text { formas más responsables de hacer y concebir el } \\
\text { turismo e iniciar formalmente el uso de la deno- } \\
\text { minación turismo sostenible. }\end{array}$ \\
\hline $\begin{array}{l}\text { Código ético mun- } \\
\text { dial para el turismo }\end{array}$ & $\begin{array}{l}\text { Asamblea General } \\
\text { de la OMT, 1999 }\end{array}$ & OMT, ONU & $\begin{array}{l}\text { Santiago de de } \\
\text { Chile, Chile }\end{array}$ & $\begin{array}{l}\text { Definir los principios del ejercicio ético de la ac- } \\
\text { tividad turística e impulsar la adhesión internacio- } \\
\text { nal a su texto, cuyo contenido fue asumido por la } \\
\text { ONU en 2002. }\end{array}$ \\
\hline
\end{tabular}

Declaración de Quebec sobre el ecoturismo
Cumbre Mundial del Ecoturismo, 2002
PNUMA, OMT
Quebec, Canadá
Establecer un documento preparatorio para la Cumbre Mundial para el Desarrollo Sostenible celebrada en Johannesburgo en 2002 sobre el ecoturismo y su vínculo con el desarrollo sostenible.
Declaración de Ciudad del Cabo sobre el turismo responsable
Conferencia sobre turismo responsable en destinos. Ciudad del Cabo, 2002
Centro Interna-

cional de Turismo Ciudad del

Responsable y Cabo, Sudá-

Turismo de El frica

Cabo Occidental
Dar formato de alcance internacional al trabajo previo de Sudáfrica para la formulación de las directrices del turismo responsable en un acto paralelo a la Cumbre Mundial para el Desarrollo Sostenible celebrada en Johannesburgo en 2002. 
Tabla 01 - Características de los documentos internacionales analizados.

\begin{tabular}{lllll}
\hline \multicolumn{1}{c}{ Fuente } & \multicolumn{1}{c}{ Evento } & Promotores & Locación & \multicolumn{1}{c}{ Objetivo } \\
\hline $\begin{array}{l}\text { Carta de Málaga } \\
\text { sobre turismo justo }\end{array}$ & $\begin{array}{l}\text { Encuentro Interna- } \\
\text { cional sobre Turismo } \\
\text { Justo, 2006 }\end{array}$ & $\begin{array}{l}\text { Diputación de } \\
\text { Málaga con } \\
\text { apoyo de la OMT } \\
\text { y EuroCOTAL }\end{array}$ & $\begin{array}{l}\text { Málaga, Anda- } \\
\text { lucía, España }\end{array}$ & $\begin{array}{l}\text { Lograr la adopción de compromisos de los orga- } \\
\text { nismos multilaterales y las administraciones cen- } \\
\text { trales, regionales y locales, los agentes e interme- } \\
\text { diarios turísticos, las comunidades locales, las or- } \\
\text { ganizaciones sociales y las universidades para el } \\
\text { desarrollo del turismo justo. }\end{array}$ \\
\hline
\end{tabular}

Declaración de Muscat sobre turismo y cultura
II Conferencia Mundial sobre Turismo y OMT, UNESCO Cultura, 2017
Emitir una declaración para impulsar el desarroMuscat, Sulta- llo sostenible mediante el turismo y la cultura, en nato de Omán el marco del Año Internacional del Turismo Sostenible para el Desarrollo.

Fuente: Elaboración propia (2019).

\section{Gobernanza de la gestión}

La gobernanza puede ser definida como las interacciones entre las estructuras, procesos y tradiciones que determinan qué poder y responsabilidades son ejercidas, cómo se toman las decisiones y cómo participan los ciudadanos y otras partes interesadas (Graham et al., 2003). La gobernanza incluye la participación de actores en las decisiones que puedan afectarlos, consensos informados, información de apoyo a la toma de decisiones y registro de las decisiones y acciones para su implementación (Borrini-Feyerabend et al., 2013). Asimismo, Pazmiño (2013) indica que la gobernanza es la relación articulada que mantienen actores, normas e instituciones.

\section{Conservación del patrimonio}

La UICN (1980) planteó los tres objetivos fundamentales para conservar los recursos vivos del planeta: mantener los procesos ecológicos esenciales y los sistemas vitales, preservar la diversidad genética y asegurar el aprovechamiento sostenido de las especies y de los ecosistemas. En el presente estudio se ha analizado cómo el turismo puede contribuir en la búsqueda de alcanzarlos o, por lo contrario, está siendo un obstáculo.

\section{Identidad cultural y autenticidad}

Identidad cultural y autenticidad: Este criterio es recurrente en los documentos internacionales analizados. El vínculo entre el turismo y estos dos conceptos es indesligable. El tema de identidad está bien definido por Molano (2007), quien señala que "el concepto de identidad cultural encierra un sentido de pertenencia a un grupo social con el cual se comparten rasgos culturales, como costumbre, valores y creencias. La identidad no es un concepto fijo, sino que se recrea individual y colectivamente y se alimenta de forma continua de la influencia exterior". El sentido de pertenencia cultural puede vincularse tanto al patrimonio cultural tangible como al intangible. Por otro lado, lo Consejo Internacional de Monumentos y Sitios (ICOMOS, 1994), en la Conferencia de Nara sobre autenticidad, señala que el reconocimiento de la autenticidad juega un papel importante en todos los estudios científicos del patrimonio cultural, en los planes de conservación y restauración, así como en el procedimiento de inscripción en la Lista del Patrimonio Mundial y otros inventarios de patrimonio. Estos dos conceptos tienen en común la valorización de la cultura local, la importancia de los estudios científicos, de las fuentes de información y la apropiación e identificación benéfica de la población hacia su riqueza ancestral.

\section{Equidad e inclusión}

Equidad e inclusión: Se busca relacionar ambos términos con el turismo, intentando que la población local que vive dentro del área natural protegida o en su zona de amortiguamiento tenga el derecho y la oportunidad de ser tratados bajo esas condiciones. La equidad social - en el contexto de las iniciativas de conservación y desarrollo sostenible - es un conjunto de prácticas tendientes al abordaje y superación de todas las formas sociales, económicas, culturales y políticas de exclusión e inequidad. Para el efecto se proponen mecanismos concretos de redistribución de la riqueza, los recursos y las oportunidades, así como la construcción de un verdadero balance intercultural y de género en la toma de decisiones relacionadas con proyectos y políticas en este ámbito (UICN, 2019). El Ministerio de Desarrollo e Inclusión Social (MIDIS, 2013) del Perú define "inclusión social" como la situación en la que todas las personas puedan ejercer sus derechos, aprovechar sus habilidades y tomar ventaja de las oportunidades que se encuentran en su medio. 


\section{Mercado y comercialización}

Para la American Marketing Association (AMA, 2013) mercadeo o mercadotecnia es "la actividad, el conjunto de instituciones y los procesos para crear, comunicar, entregar e intercambiar ofertas que tienen valor para los clientes, clientes, socios y la sociedad en general. Aunque esta definición sigue vigente en el mundo del mercadeo, un elemento clave ha surgido en los últimos años que está revolucionando la aproximación de las empresas con los clientes y potenciales clientes: los medios digitales y las redes sociales. En este sentido, Kotler e Armstrong (2007), argumentan que el uso extendido de internet y otras nuevas tecnologías están teniendo un impacto drástico tanto para los vendedores como para los compradores, considerando que los vendedores deberán desarrollar nuevas estrategias y prácticas adecuadas al nuevo entorno.

La comercialización, por su parte, siempre se ha tratado como la acción de comercializar. La Secretaría de Economía de México (2010) considera a la comercialización como el conjunto de acciones y procedimientos para introducir eficazmente los productos en el sistema de distribución, organizando su posicionamiento y encontrándole la forma que los vuelvan atractivos en el mercado. En el caso de las áreas naturales protegidas, si bien el consumidor tiene que desplazarse para hacer uso del recurso, es importante definir qué se debe hacer para posicionarlas y lograr el consumo del visitante.

\section{Experiencia del visitante}

El turismo, especialmente en un espacio natural protegido, debe gestionarse de forma sostenible. Una gestión sostenible maximiza los beneficios que la actividad turística genera en el territorio de un espacio natural protegido y minimiza los daños que podría ocasionar si se desarrolla sin planificar y de forma poco respetuosa, amenazando con ello los valores naturales y culturales de estos espacios, que son, a la postre, los principales atractivos turísticos (Gómez-Limón et al., 2010). Esa gestión no solo debe incluir la cautelación de los atractivos turísticos, sino satisfacer las expectativas de los visitantes, tomando en cuenta, tal como plantea la Organización Mundial de Turismo (2010), que esta tarea es responsabilidad tanto de los funcionarios públicos como de los empresarios y otros actores involucrados en el producto.

El manejo de visitantes debe basarse en varios principios fundamentales: (i) entender que las características del visitante, sus motivaciones y expectativas son fundamentales para tener políticas efectivas de manejo; (ii) las instalaciones relacionadas con los visitantes generalmente representan tanto las mejores oportunidades para apreciar las áreas como las amenazas internas a su integridad biofísica y cultural; (iii) mientras que el turismo es una industria dirigida por el mercado, el manejo de las áreas protegidas está determinado por mandatos legislativos; (iv) los impactos negativos por uso de los visitantes siguen patrones predecibles que pueden usarse para sistemas de gestión de la estructura $\mathrm{y}$ acciones (Eagles e McCool, 2003).

\section{Resultados del análisis de las entrevistas a pro- fundidad}

De las 24 entrevistas realizadas a los especialistas, se pudo obtener una serie de comentarios y sugerencias que sirvieron para diseñar las variables a incorporar en los seis criterios determinados en la etapa anterior y para construir la matriz de evaluación. Entre los principales están:

- El turismo en las áreas naturales protegidas debe ser una actividad complementaria a la misión de las áreas naturales protegidas: la conservación del patrimonio natural y cultural que alberga, incluyendo la participación activa de la población local que preserve su relación con el entorno y su economía tradicional.

- El turismo, con todo el potencial para generar ingresos, pero al mismo tiempo la posibilidad de impactar los objetos de conservación del área, debe ser ordenado y regulado.

- Contar con documentos oficiales de planificación del turismo y que se trabaje con indicadores de logros.

- Las áreas deben contar con personal especializado o al menos entrenado en aspectos relacionados a la planificación turística y el manejo del área para la experiencia del visitante.

- La jefatura debe ser proactiva en cuanto al fomento de la actividad turística en el área.

- Los comités de gestión de las áreas deben tener como prioridad en su gestión el apoyo al turismo.

- Es preciso aprovechar los valores naturales, arqueológicos y culturales de las áreas.

- Resulta indispensable desarrollar estrategias eficientes de mercadeo, especialmente en las redes sociales, así como de interpretación del patrimonio.

- Debe fortalecerse a los guías y orientadores locales mediante la capacitación y actualización de información sobre las investigaciones por las instituciones involucradas en el área.

- Es necesario reforzar el vínculo entre la autoridad del área y las instituciones públicas que hacen investigación y otras actividades en el lugar. 
- Para aumentar la demanda, se debe diversificar la oferta turística dentro del área,

- Es importante que los operadores estén ofreciendo actividades diferenciadas, como por ejemplo, deportes de aventura.

- El turismo debe ser un vehículo para fortalecer la identidad local con su legado natural e histórico.

- El área debe participar en las plataformas regionales con respecto al desarrollo del turismo y tener vínculos con otros atractivos regionales.

- Es preciso reducir los trámites burocráticos en cuanto a iniciativas locales de emprendimientos turísticos.

- Resulta necesario considerar la pertinencia de que los visitantes sean obligatoriamente acompañados con un guía u orientador.

- El cobro de entrada a los visitantes debe estar en función de los servicios que se les brindan.

- El área debe contar con iniciativas novedosas que involucren a la población local en la actividad turística.

- Las asociaciones locales vinculadas con el turismo deben tener mayor voz.

- Hace falta fomentar la presencia de la cooperación internacional y nacional para la promoción turística del área.

- Se debe usar la tecnología para mejorar la experiencia, como por ejemplo APP, GPS, reportes en línea, mapas interactivos, ubicación de especies encontradas, entre otros.

- Es necesario habilitar instalaciones para la mejor experiencia del visitante: centro de visitantes, senderos, señalización, puntos de venta de alimentos y bebida, miradores, entre otros.

\section{Diseño preliminar de la matriz de evaluación}

Para cada uno de los seis criterios estipulados en las dos primeras etapas de la investigación, se tomaron en consideración los temas abordados en cada uno de los documentos internacionales, las opiniones y comentarios de los entrevistados y la revisión bibliográfica, se atribuyeron variables a cada uno de los criterios, con un total de 62 variables que se discutieron en el taller de validación.

\section{Validación de la matriz de evaluación}

Para validar la propuesta de matriz de evaluación del manejo del turismo en áreas naturales protegidas se realizó lo siguiente: se seleccionaron 14 especialistas en los temas de turismo, áreas naturales protegidas, planificación y servicios turísticos. Con ese fin, los investigadores revisaron hicieron consultas con personas clave y revisaron los trabajos realizados por los candidatos. Se realizó la invitación por correo o por teléfono a los especialistas, señalándose el lugar, día y hora donde se realizaría el taller. Cuando los participantes confirmaron su presencia, se procedió a enviarles materiales para la familiarización previa. Los invitados fueron representantes del sector público, privado y académico. El taller comenzó con una explicación del estudio que se estaba desarrollando y con la presentación de 62 variables propuestas inicialmente, con las alternativas de puntaje para cada una de ellas. Se planteó un ejercicio en el que se fueron discutiendo cada variable y sus niveles de alternativa, y al acabar la revisión de cada uno de los seis criterios, se les pidió que anotaran sus comentarios y sugerencias. El taller duró cerca de tres horas, se redujeron las variables a 56 y se obtuvo material relevante para contar con la versión final de la matriz.

\section{Versión final de la matriz de evaluación del ma- nejo del turismo en áreas naturales protegidas}

A partir de las fases anteriores, se diseñó la versión final de la matriz de evaluación del manejo del turismo en áreas naturales protegidas, con seis criterios generales y 56 variables, repartidos según las siguientes cifras: gobernanza de la gestión: 6 variables; conservación del patrimonio: 6 variables; identidad cultural y autenticidad: 4 variables; inclusión y equidad: 5 variables; mercadeo y comercialización: 8 variables, y experiencia del visitante: 27 variables. El alto número de variables para el criterio "experiencia del visitante" hizo que fuese dividido en tres grupos: accesibilidad y servicios de llegada al área, exploración y aprendizaje, y protocolos y servicios de apoyo a los visitantes.

Cada una de estas variables contó con cuatro niveles de avance diferenciado, con el fin de ayudar al evaluador a definir mejor el grado de avance obtenido en cada una de las variables. Por efectos de espacio, en el presente artículo no se presentan las alternativas planteadas para cada variable, sino solo los espacios para el puntaje de cada variable: (i) gobernanza de la gestión: instrumentos oficiales de gestión, priorización del turismo, efectividad de zonificación, eficiencia del personal, participación de gestores culturales y participación del comité de gestión en turismo; (ii) conservación del patrimonio: inventario de biodiversidad, de sitios arqueológicos, de manifestaciones de cultura viva, monitoreo de recursos turísticos naturales, de recursos turísticos arqueológicos, participación del comité de gestión en conservación; (iii) identidad cultural y autenticidad: manifestaciones culturales ancestrales, uso económico y productivo de los recursos, material informativo sobre valores naturales y culturales, souvenirs con identidad local; (iv) inclusión 
y equidad: pobladores locales participan en turismo, empresas contratan personal local, mecanismos de apoyo a comunidades, capacitación en turismo brindado para población local, involucramiento de personas con discapacidad; (v) mercadeo y comercialización: actividad de operadores turísticos, prioridad del área en el destino, información sobre la demanda en el área, información general del área en medios digitales, información turística del área en medios digitales, registro de prestadores de servicios en el área, datos sobre visitantes y medición de satisfacción del visitante; (vi) experiencia del visitante: accesibilidad y servicios de llegada al área (alternativas de acceso al área, estado del camino principal al área, caminos alternativos de acceso al sitio, avisos u otros indicadores de llegada al sitio, estacionamiento, sistema de boletaje para el expendio de entradas, área de recepción del visitante, servicios higiénicos en la zona de recepción, código de conducta básico del visitante, centro de interpretación y servicio de alquiler de equipos); exploración y aprendizaje (panel directorio informativo del área, servicio de guías u orientadores locales, senderos para los visitantes, señales en los senderos y los atractivos, instalaciones de apoyo a actividades de turismo, recursos interpretativos en el campo, servicios higiénicos próximos a atractivos principales, sistema de disposición de residuos sólidos, cafeterías y puntos de venta de alimentos y bebidas y tienda de souvenirs) y protocolos y servicios de apoyo al visitante (acceso a señal telefónica e internet, protocolos para la seguridad del visitante, protocolos de atención de emergencias, protocolos para la gestión de la carga de visitantes, protocolos para la gestión de residuos sólidos y sistema de atención a visitantes).

A cada una de las 56 variables se le asignaron cuatro niveles de cumplimiento, a los cuales debía asignarse un puntaje según el grado de avance obtenido, buscándose que las cuatro alternativas sean lo más objetivas posibles para facilitar la evaluación de los criterios y variables. Se diseñó un sistema de asignación de puntajes a cada variable de cada criterio con una escala de cuatro puntos $(0,1$, 2 y 3). La intención fue que el evaluador definiese el nivel de progreso de cada variable: 0 cuando no ha habido ningún avance o este ha sido mínimo; 1 cuando ha existido algún progreso; 2 cuando hay un avance significativo, pero aún puede mejorarse, y 3 cuando se ha alcanzado o está cerca de alcanzarse la situación óptima. La tabla 2 puede usarse como referencia.

Tabla 02 - Escala de calificación de las variables evaluadas.

\begin{tabular}{ccc}
\hline \% del óptimo & Avance de la variable & Puntaje \\
\hline $0-35$ & Insatisfactorio & 0 \\
$36-60$ & Poco satisfactorio & 1 \\
$61-80$ & Medianamente satisfactorio & 2 \\
$81-100$ & Satisfactorio - muy satisfactorio & 3 \\
\hline
\end{tabular}

Fuente: Adaptado de Cifuentes et al. (2000)

Como los seis criterios generales no tienen el mismo número de variables que ayuden a analizarlo, para cada uno de los criterios se aplicó la siguiente fórmula:

$$
\text { Puntaje del criterio }=((\mathrm{X} 1+\mathrm{X} 2 \ldots+\mathrm{Xn}) / 3 * n) * 100
$$

Donde:

$\mathrm{X}=$ Puntaje de la variable

$\mathrm{N}=$ Número de variables del criterio

Cada criterio tendrá entonces un puntaje (la suma de puntos de cada variable dividida entre el número de variables multiplicado por tres, multiplicado por 100. El puntaje obtenido por cada criterio se compara con los rangos de la tabla 2 y se obtiene el nivel de avance de cada criterio. Para la evaluación del área general, se suman los promedios de los seis criterios, se divide entre seis y se obtendrá un puntaje que será contrastado con la matriz, que indicará el grado global del avance del manejo del turismo en el área. Sin embargo, más allá del indicador global, es preciso encontrar las variables que están con menores avances y aquellas que han llegado o están próximas al nivel óptimo. Con esta finalidad se puede usar un gráfico "radar" o "telaraña" (figura 2) para apreciar visualmente aquellas variables que tienen menores avances. La Sociedad Latinoamericana para la Calidad (2000) señala "el radar" como una de las mejores herramientas para mostrar visualmente las brechas entre el estado actual y la situación ideal. Al obtenerse los puntajes promedio de los seis criterios, se aplican los resultados a una gráfica de radar comparativa, esta vez entre los seis criterios. Con esto y con los promedios de cada criterio, puede compararse cuáles de estos están con mayor o menor avance y por lo tanto requieren mayor esfuerzo de trabajo e inversión. 


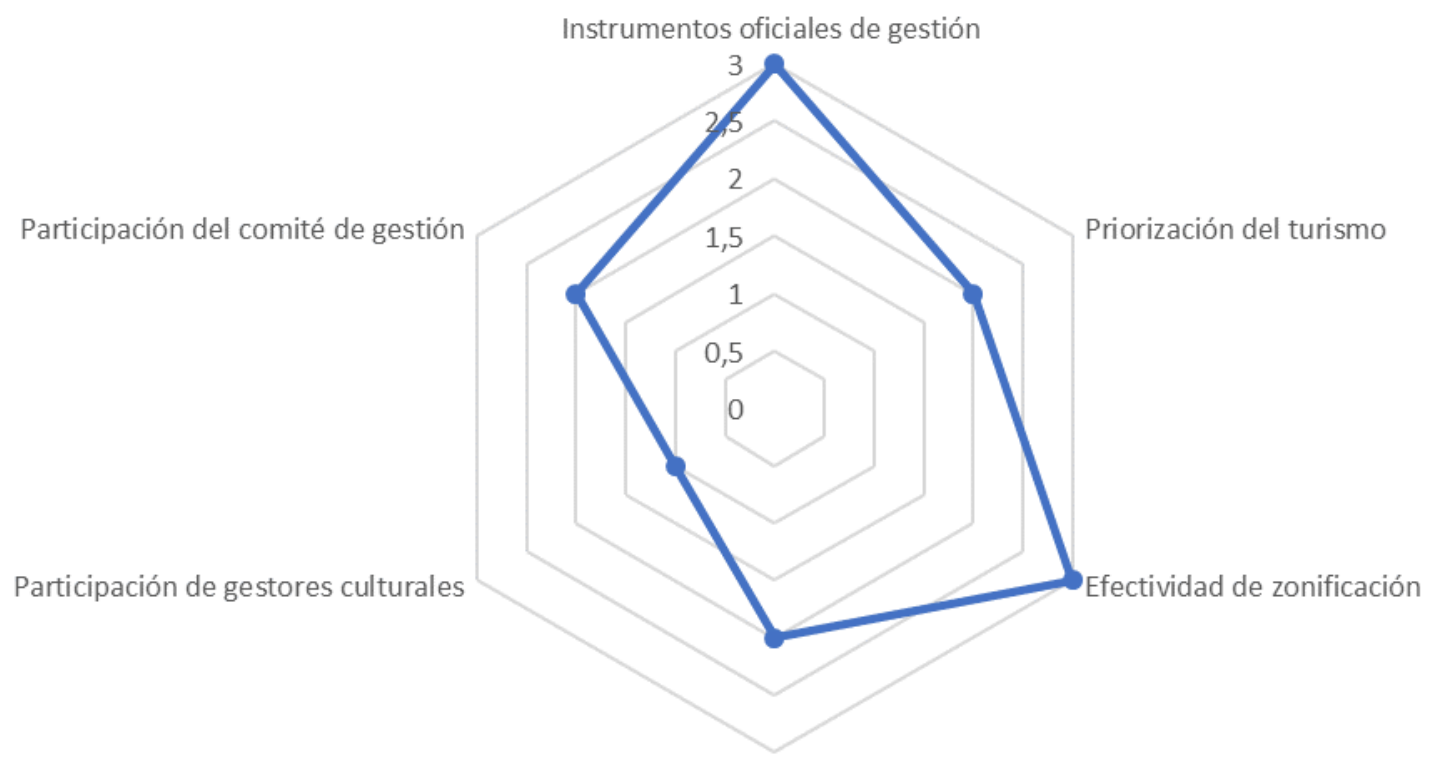

Eficiencia del personal

Fuente: Elaboración propia (2019)

Figura 02 - Ejemplo de uso de gráfico radar para la visualización del avance en un criterio.

En el ejemplo de la figura 2, se puede apreciar que la participación de los gestores culturales y la participación del comité de gestión son las variables que requieren de mayor fortalecimiento, mientras que los instrumentos oficiales de planificación y la efectividad de la zonificación son los que han logrado mayores avances.

La información obtenida de las cifras y los gráficos obtenidos permitirá redactar el diagnóstico acerca del avance logrado por el área natural protegida en relación al turismo, destacándose los logros obtenidos y las deficiencias que deben enfrentarse, haciéndose sugerencias para la toma de decisiones de los gestores a cargo del área.

\section{CONCLUSIONES}

Si bien existen diversas propuestas metodológicas para evaluar la gestión de las áreas protegidas, esta es una propuesta novedosa enfocada específicamente a evaluar el manejo del turismo en áreas naturales protegidas individuales, e incluso puede adaptarse a otros ámbitos como sitios arqueológicos, áreas de recreación privadas o de turismo rural.

La evaluación de los documentos internacionales analizados en la investigación muestra que la gobernanza de la gestión, la conservación del patrimonio, la identidad cultural y autenticidad, la inclusión y equidad, el mercadeo y la comercialización y la gestión de la experiencia del visitante son los criterios más frecuentes a considerar dentro de lo que significa el manejo del turismo en las áreas naturales protegidas.
El uso de una matriz de evaluación permite sistematizar en mejor medida los elementos que deben ser analizados para hacer el diagnóstico de los avances realizados en el área de turismo en las áreas naturales protegidas.

Cada criterio identificado para analizar tiene sus propias características y dinámicas, por lo que no se recomienda plantear un número estándar de variables para cada uno de ellos. El uso de promedios ayuda a trabajar cada criterio independientemente.

La asignación de cuatro niveles de puntaje para indicar el grado de avance de cada variable, a lo que se agrega el uso de gráficos "radar", ayuda a visualizar en forma simple lo que se ha conseguido en cada uno de los criterios determinados para la evaluación.

\section{REFERÊNCIAS BIBLIOGRÁFICAS}

ABRAMS, P.; BORRINI-FEYERABEND, G.; GARDNER, J.; HEYLINGS, P. Evaluating governance: a handbook to accompany a participatory process for a protected area. Quebec: Parks Canada and Tilcepa-Theme on Indigenous and Local Communities, Equity and Protected Areas of IUCN CEESP/WCPA, 2003. 120p.

American Marketing Association (AMA). Definitions of marketing. 2013. Disponible en:

$<$ https://www.ama.org/the-definition-of-marketing/>. Acceso: 2 de julio del 2019.

BIESEK, A. Turismo de base comunitária e desenvolvimento territorial: políticas e práticas em Foz do Iguaçu e região. Ano de obtenção: 2013. 331p. Tese (Doutorado em Geografia) - Universidade Federal do Paraná, Curitiba. 
BORRINI-FEYERABEND, G.; DUDLEY, N.; JAEGER, T.; LASSEN, B.; PATHAK BROOME, N.; PHILLIPS, A.; SANDWITH, T. Governance of protected areas: from understanding to action. Best Practice Protected Area Guidelines, Series n.20, Gland, Switzerland: IUCN, xvi, 2013. $124 \mathrm{p}$.

CHIRINO, J.; ABAD, J.; BELLOT. Uso de indicadores de Presión-Estado. Respuesta en el diagnóstico de la comarca de la Marina Baixa, SE, España. Ecosistemas, v.17, n.1, p.107-114, 2008.

CIFUENTES, M.; IZURIETA, A.; HENRIQUE DE FARIA, H. Medición de la efectividad del manejo de áreas protegidas. Turrialba, Costa Rica: WWF, IUCN, GTZ, 2000. 105p.

Conferencia Mundial de Turismo Sostenible. Carta del Turismo Sostenible. Lanzarote, Islas Canarias, España, 1995, 8 p. Disponible en: 〈http://www.icomoscr.org/doc/teoria/VARIOS.1995.carta.lanzarote.turismo.sostenible.pdf $>$. Acceso: 2 de febrero de 2019

EAGLES, P.F.J.; MCCOOL, S.F. Tourism in national parks and protected areas: planning and management. CABI, UK, 2003. 320p.

Encuentro Internacional Sobre Turismo Justo. Carta de Málaga sobre el turismo justo. Málaga, s/p, 2006. Disponible en: <http://www.upv.es/contenidos/CA-

MUNISO/info/U0579246.pdf>. Acceso 14 de enero del 2019.

GÓMEZ-LIMÓN, J.; GUZMÁN, A.; DE ANDRÉS, A. Guía para la adhesión de las empresas turísticas a la Carta Europea de Turismo Sostenible en espacios protegidos. EUROPARC - España, 188p. 2010.

GRAHAM, J.; BIEN, A.; PLUMPTRE, T. Governance principles for protected areas in the 21 st century. Fifth World Parks Congress, South Africa in September 2003. Ottawa, 2003. 40p.

HOCKINGS, M.; STOLTON, S.; LEVERINGTON, F.; DUDLEY, N.; COURRAU, J. Evaluating effectiveness: a framework for assessing management effectiveness of protected areas. 2nd Edition. Gland, Suiza y Cambridge, Reino Unido: IUCN, 2006. 105p.

HOCKINGS, M.; STOLTON, S.; COURRAU, J.; DUDLEY, N.; PARRISH, J.; JAMES, R.; MATHUR, V.; MAKOMBO, J. The world heritage management effectiveness workbook: 2007 Edition. UNESCO, IUCN, University of Queensland. The Nature Conservancy, 2007. 106p.

ICOMOS. Conferencia de Nara sobre autenticidad. 1994. Disponible en: <http://www.icomoscr.org/doc/teoria/DOC.1994.nara.documento.sobre.autenticidad.pdf >. Acceso: 4 de marzo de 2019.

International Conference on Responsible Tourism in Destinations. The Cape Town Declaration. Cape Town, 2002. S/P.

KOTLER, P.; ARMSTRONG, G. MARKETING. Versión para Latinoamérica. Decimoprimera edición. Pearson Educación México. 2007. 706p.

LEUNG, Y. F.; SPENCELEY, A.; HVENEGAARD, G.; BUCKLEY, R. (eds.). Gestión del turismo y de los visitantes en áreas protegidas: directrices para la sostenibilidad. Serie Directrices sobre Buenas Prácticas en Áreas Protegidas n.27, Gland, Suiza: IUCN. 2019, 120p.
LEVERINGTON, F.; LEMOS COSTA, K.; COURRAU, J.; PAVESE, H.; NOLTE, C.; MARR, M.; COAD, L.; BURGESS, N.; BOMHARD, B.; HOCKINGS, M. Management effectiveness evaluation in protected areas: a global study. Second edition; Australia: The University of Queensland Brisbane, 2010. 101p.

Ministerio de Desarrollo e Inclusión Social (MIDIS). Estrategia nacional de desarrollo e inclusión social. 2013. Lima. $92 \mathrm{p}$.

Ministerio del Ambiente del Ecuador. Evaluación de efectividad de manejo del patrimonio de áreas naturales del estado. Guía Metodológica. Quito, 2014. 85p.

MOLANO, O. Identidad cultural un concepto que evoluciona. Revista Opera, Bogotá, n.7, p.69-84, 2007.

Organización Mundial del Turismo; Sultanato de Omán; Organización de las Naciones Unidas para la Educación, Ciencia y la Cultura. Muscat declaration on tourism and culture: fostering sustainable development. Second UNWTO/UNESCO World Conference on Tourism and Culture: Fostering Sustainable Development. Muscat, 2017. $4 \mathrm{p}$.

Organización Mundial del Turismo (OMT). Normas y sistemas de calidad en el turismo y su relación con la sostenibilidad y las leyes de turismo: la experiencia de las Américas. Madrid, 2010. 138p.

Organización Mundial del Turismo. Código ético mundial para el turismo. Resolución de la Asamblea General de la OMT en Santiago, 1999, Madrid, 8p. Disponible en: $<$ http://www.ugto.mx/images/eventos/06-07-16/codigoetico-mundial-turismo.pdf $>$. Acceso: 28 de enero de 2019.

PAZMIÑO, A. Aprendiendo sobre gobernanza. Conservación Ahora, publicación electrónica de la Oficina Regional para América del Sur de la UICN, 2013. Quito, p.17-19.

Programa de las Naciones Unidas para el Medio Ambiente; Organización Mundial del Turismo. Declaración de Quebec sobre el ecoturismo. Cumbre Mundial del Ecoturismo, 2002. 9p. Disponible en: <http://www.turismoycooperacion.org/OBSERTUR/X/03.pdf>. Acceso: 14 de enero de 2019.

RIVERO BLANCO, C; GABALDÓN, M. Evaluación de sistemas de áreas naturales protegidas: una metodología numérica. Parques, v.3, n.1, p.11-13, 1992.

RODRÍGUEZ, T. Convenios internacionales y ambiente: recomendaciones para una mejor aplicación de los convenios seleccionados en los países de la iniciativa del corredor del pacífico este tropical. $1^{\mathrm{a}}$ ed. San José, C.R.: Fundación Marviva, 2009. 102p.

SÁNCHEZ-CORTES, J.L.; ARREDONDO-GARCÍA, M.; LEYVA-AGUILERA, J. ÁVILA-SERRANO, G.; FIGUEROA-BELTRÁN, C; MATA-PERELLÓ, J. Propuesta de matriz para evaluación de proyectos geoparques en América Latina, con base en Áreas Naturales Protegidas: Aplicación y Casos de Estudio. Pasos. Revista de turismo y patrimonio cultural, v.12, n.2, p.383-394, 2014.

SANCHO, A.; GARCÍA, G.; ROZO, E. Comparativa de indicadores de sostenibilidad para destinos desarrollados, en desarrollo y con poblaciones vulnerables. Annals of Tourism Research, v.9, n.1, p.150-177, 2007. 
Secretaria de Economía de México. México Emprende. 2010. Disponible en: <http://www.2006-2012.econo-

mia.gob.mx/mexico-emprende/productos-servicios/comercializacion>. Acceso: 27 de febrero de 2019.

Sociedad Latinoamericana para la Calidad. Gráfica de radar. 2000. Disponible en: <http://www.educarchile.cl/UserFiles/P0001/File/radar.pdf>. Acceso: 17 de marzo de 2019.

STOLTON. S.; HOCKINGS, M.; DUDLEY, N.; MACKINNON, K.; WHITTEN, T.; LEVERINGTON, F. The management effectiveness tracking tool (METT). Gland, Suiza: WWF Internacional, 2007, 22p.

UICN. Equidad social. Portal sobre conservación y equidad social de la UICN. 2019. Disponible en: <https://www.portalces.org/biblioteca>. Acceso: 12 de febrero de 2019.

UICN. Estrategia mundial para la conservación. La conservación de los recursos vivos para el logro de un desarrollo sostenido. UICN, PNUMA y WWF. Gland. 1980. 71 p.

WEISKOPF, B.; LANDERO, M. Guía metodológica para la implementación talleres fomento cadenas de valor. GTZ Nicaragua. Programa Manejo Sostenible de Recursos Naturales y Fomento de Competitividad (MASRENACE). Equipo Regional de Competencias Cadenas de Valor y PPP (ERC). Ciudad de Guatemala, 2009. 62p.

WORBOYS, G.L.; LOCKWOOD, M.; KOTHARI, A.;

FEARY, S.; PULSFORD, I. (eds.). Protected area governance and management. Canberra: ANU Press, 2015. 966p. 